\title{
DERIVATION OF CURRENTS AND DIAMAGNETIC EFFECTS FROM GLOBAL PLASMA PRESSURE DISTRIBUTIONS OBTAINED BY IMAGE/HENA
}

\author{
E. C. Roelof, P. C:son Brandt and D. G. Mitchell \\ The Johns Hopkins University Applied Physics Laboratory, Laurel, MD
}

\begin{abstract}
The global ion distributions inverted from ENA images measured by IMAGE/HENA can be used to calculate the plasma pressure. In principle, knowing the plasma pressure distribution and the magnetic field, one can also calculate the three-dimensional current system that is driven by the pressure gradient. The currents perpendicular to the magnetic field depend on the pressure gradient and the magnetic field strength. Assuming current continuity $(\nabla \cdot \mathbf{J}=0)$, the parallel currents are driven by the divergence of the perpendicular current. We will describe a method where we use Euler potentials to calculate the current distribution. The method will then be applied to some pressure distributions measured by IMAGE/HENA and the resulting field aligned currents (FAC) will be compared to the global FAC maps derived from Irridium magnetometer data.
\end{abstract}

Key words: Ring current, region 2 current system, ENA imaging

\section{GLOBAL PRESSURE DISTRIBUTION}

ENA images from HENA are inverted using a constrained linear inversion developed by Demajistre et al. (2002) that has been described by C:son Brandt et al. (2002a) to obtain the distribution function of protons, and from there compute the partial pressure. In the current algorithm this is done by assuming a dipole field and isotropic pitch-angle distributions.

Figure 1 shows the equatorial proton pressure contours thus derived during the main phase of the 4 October 2000 storm. Arrows are the computed equatorial currents (see more below). We note that the peak of the pressure occurs in the post-midnight sector. This is a common feature of the main phase proton ring

Preprint submitted to Elsevier Science 28 October 2002 


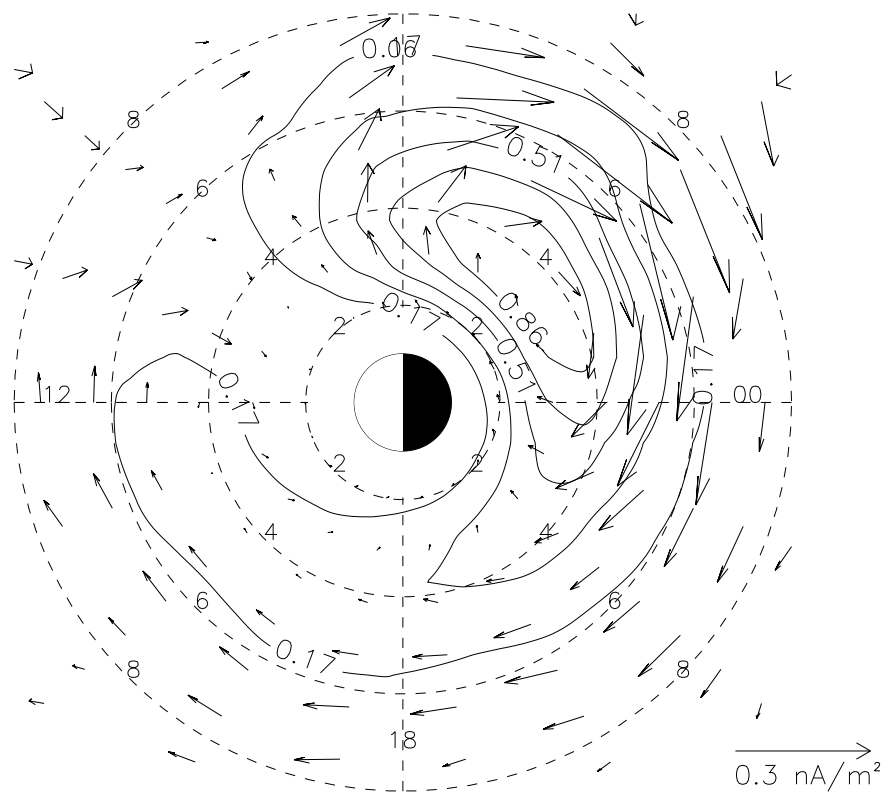

Fig. 1. The pressure contour map from the deep main phase of the 4 October 2000 storm at 18:00 UT for 27-60 keV. Arrows represent the equatorial current densities. The example arrow represents $0.3 \mathrm{nA} \mathrm{m}^{-2}$.

current (C:son Brandt et al., 2002b). Also note that the peak occurs as deep as $\mathrm{L}=3-4$. We expect that this basic information will prove valuable in initial estimates of how significant the ring current effects are on the geomagnetic field.

The collected pressure distributions for different storms will be an important characterization of the storm-time ring current for comparisons with magnetospheric models (C:son Brandt et al., 2002b). More specifically, the global pressure gradients drive the region 2 currents and their closure in the ionosphere. This current system is a vital component in determining the storm-time ionospheric electric field. These ionospheric electric fields are known to lift up and diminish the ionospheric F-region plasma during severe storms (Foster and Rich, 1998), which is the main space-weather disturbance for ground communication systems.

Moreover, the pressure datasets can readily be used to compute the Dst via the Dessler-Parker-Sckopke (DPS) relation. This is important in finding how much of the Dst index can be attributed to current systems other than the ring current (Ohtani et al., 2001). 


\section{GLOBAL RING CURRENT INTENSITY}

Having the global pressure distribution it is possible to derive the perpendicular currents $\mathbf{J}$ from

$$
\mathbf{J} \times \mathbf{B}=\nabla \cdot \mathbf{P}
$$

where $\mathbf{P}$ is in general the pressure tensor and $\mathbf{B}$ is the magnetic field. By assuming a divergence free current $(\nabla \cdot \mathbf{J}=0)$ one can also compute the fieldaligned components of the current. The 3D current system has been computed by utilizing an Euler potential formalism for $\mathbf{J}$ itself as described by Roelof (1989) and a dipole magnetic field. In this formulation one assumes the pressure tensor to be isotropic. This is an acceptable starting point for developing

this method and is also the underlying assumption T2000 used in including the partial ring current into his magnetic field model.

\subsection{Computing the $3 D$ current system}

With an isotropic (scalar) pressure $P$ the current can be written

$$
\mathbf{J}=\nabla Q \times \nabla P,
$$

where the Euler potential $Q$ can be written for a dipole field

$$
Q(L, \mu)=\frac{R_{E}}{B_{0}} L^{4} \mu p(\mu)
$$

where $\mu=\cos \theta$ is the cosine of the co-latitude $\theta ; B_{0}=\mu_{0} M /\left(4 \pi R_{E}^{3}\right)$, and $p(\mu)$ is a sixth degree polynomial in $\mu$. The components of the current then become

$$
\begin{aligned}
& J_{r}=-\frac{L^{2}}{R_{E} B_{0}}\left(\frac{8 \mu^{2} p(\mu)}{\sin ^{6} \theta}+\sin ^{2} \theta\right) \frac{\partial P}{\partial \phi} \\
& J_{\theta}=-\frac{4 L^{2}}{R_{E} B_{0}} \frac{\mu p(\mu)}{\sin ^{5} \theta} \frac{\partial P}{\partial \phi} \\
& J_{\phi}=\frac{L^{3} \sin ^{3} \theta}{R_{E} B_{0}} \frac{\partial P}{\partial L}
\end{aligned}
$$

The field-aligned current (FAC) depends only on the azimuthal pressure gradients, while azimuthal current component depends only on the radial pressure 


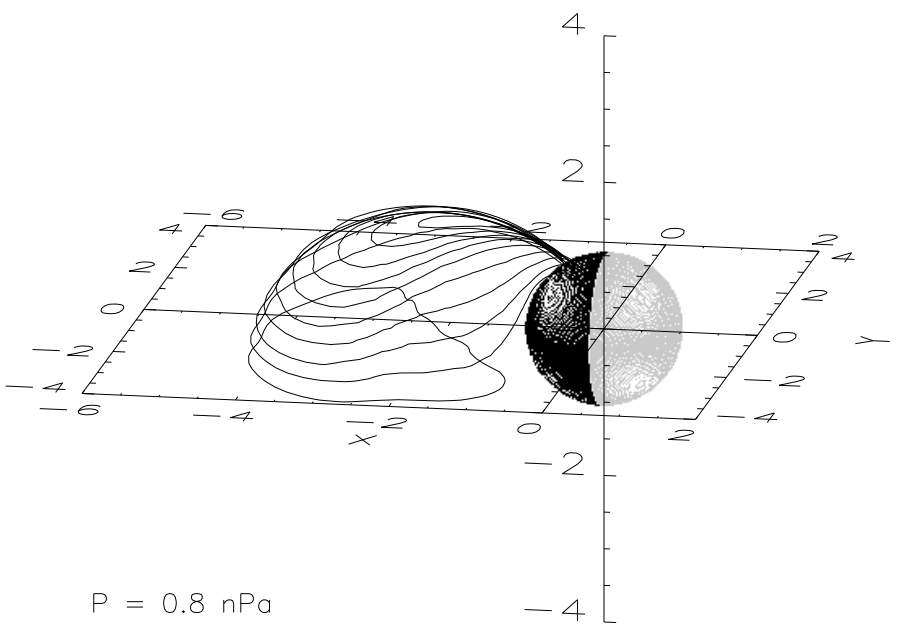

Fig. 2. Current lines obtained from the intersection of the pressure isosurface of 0.8 $\mathrm{nPa}$ and different iso surfaces of Euler potential $Q$. Each current line carries 2800 A. The viewing is from $20^{\circ}$ latitude at 07 MLT.

gradients. The equatorial currents $\left(J_{\phi}, J_{r}\right)$ are plotted as arrows in Figure 1 with the longest arrow being approximately $0.3 \mathrm{nPa} / \mathrm{m}^{2}$. The currents lying in the equatorial plane follow the pressure contours as they should.

The electrical current lines can be obtained from Equation (2) as the intersection between the surfaces of constant $Q$ and $P$, because the current intensity in Amperes is simply $I=\Delta Q \Delta P$ where $\Delta Q$ and $\Delta P$ give the spacing between the surfaces. Figures 2 and 3 show sets of electric current lines for the 27-60 $\mathrm{keV}$ proton pressure obtained at 18:00 UT on the 4 October 2000 (same as Figure 1). The current lines were obtained from the intersections between the 0.8 and $1.2 \mathrm{nPa}$ isosurface and 15 different isosurfaces of $Q$. With the chosen spacing in $Q$ each current line corresponds to a current intensity of 2800 $\mathrm{A} / \mathrm{nPa}$. This means that the total current flowing between pressure isosurfaces with a $1 \mathrm{nPa}$ spacing is $2800 \mathrm{~A}$. This means that the closer together the current lines are in space, the higher is the current density. Note that the current close to the equator does not close through the ionosphere. These figures are displayed in a format similar to Figure 10 in T2000 and show a good overall agreement, but with the peak of the pressure on lower L-shells.

To test the validity of the obtained current system we can compute the FAC going in and out of the ionosphere and compare them with the FACs obtained from the Iridium mangetometer data which is managed by Dr. Brian J. Anderson at JHU/APL. The computed FACs can also be compared to models such as the Comprehensive Ring Current Model (CRCM) (Fok et al., 2001). 


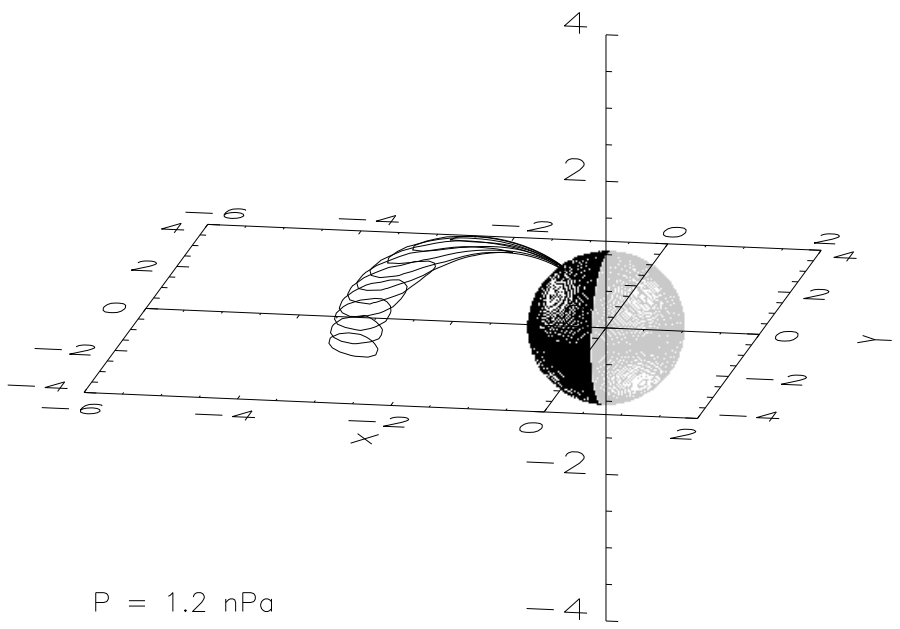

Fig. 3. Current lines obtained from the intersection of the pressure iso surface of 1.2 $\mathrm{nPa}$ and different iso surfaces of Euler potential $Q$. Each current line carries 2800 A. The viewing is from $20^{\circ}$ latitude at 07 MLT.

Some independent ways to test the validity of the near-equatorial current systems is to compute the magnetic disturbances and compare those to in-situ measurements and the T2000 model (more below).

The FAC at the surface of the Earth is to a good approximation simply the radial component of the current given by Equation (4) evaluated at $1 R_{E}$. Figure $4 \mathrm{~b}$ shows the contours of the FAC going in and out of the ionosphere for the pressure distribution shown in Figure 1. The peak values of the FACs are about $0.05 \mu \mathrm{A} \mathrm{m}^{-2}$. Figure $4 \mathrm{a}$ shows (in the same format) the corresponding FACs obtained by the magnetometers onboard the Iridium satellites (courtesy of Dr. Anderson, JHU/APL) at 18:00-19:00 UT on 4 October 2000. The grayscale extends from -0.81 (black) to $+0.81 \mu \mathrm{A} \mathrm{m}^{-2}$ (white). These measurements also include the region 1 current system above approximately $60^{\circ}$ latitude. The region 2 currents are the FACs seen below $60^{\circ}$. The latitudinal extent of our FACs obtained from the HENA agrees well with the Iridium data. The peak in the up going region of FACs on the dawn side also agrees well with the Iridium data. However, the region of down going current can be seen to be concentrated around midnight extending towards dusk in our results, while the Iridium data show that it extends clearly through dusk. We also note that our current values are about one order of magnitude less than the values in the Iridium data. We attribute these differences to the fact that we are only considering the partial pressure here. It is reasonable to expect that including the higher energy protons $(>60 \mathrm{keV})$ will extend the FACs through dusk, since higher energy ions drift further around due to the energy 


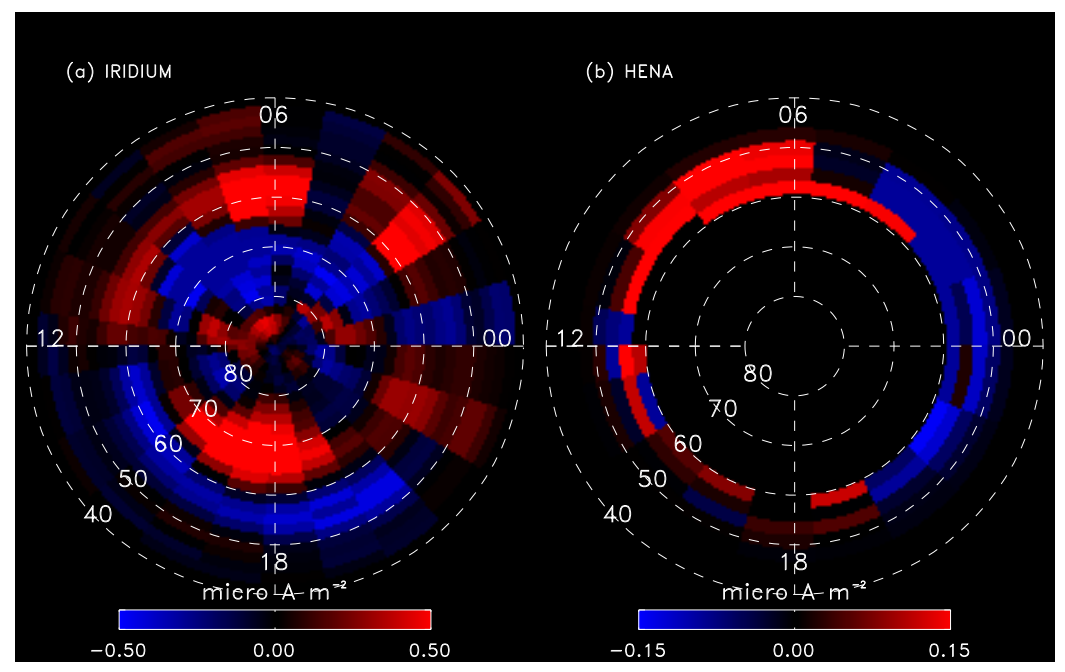

Fig. 4. (a) The FACs obtained by the magnetometer onboard the Iridium satellites at 18:00-19:00 UT on 4 October 2000. (b) The FACs going in and out of the ionosphere obtained from Equation (4) using the proton pressure distribution at 18:00 UT on the 4 October 2000 (Figure 1). Red is up going current and blue is down going.

dependence of the magnetic drift.

Figure 5 shows the azimuthal current density in the meridional at $\phi=225^{\circ}(03$ MLT), which correspond to a cut through the pressure peak in Figure 1. The figure is presented in the same format as Figure 9 in T2000. His figure shows the maximum azimuthal current to be located near the equatorial plane at around $\rho=7 \mathrm{R}_{E}$ with a peak current around $1 \mathrm{nPa} / \mathrm{m}^{2}$. Our preliminary results show an overall good agreement with the peak current located at around $\rho=6.5 \mathrm{R}_{E}$ and a lower peak current of $0.3 \mathrm{nPa} / \mathrm{m}^{2}$. Remember, however that these currents were derived from the $27-60 \mathrm{keV}$ proton pressure and therefore constitute only a partial pressure.

Given the 3D current system, one can compute the magnetic disturbances through Biot-Savart's law as was done by T2000. We will show below that it is possible to use the Euler potentials to compute the magnetic disturbance without computing the current system first. Nonetheless, we believe that visualizing the global current system directly from the ENA data gives considerable insight into the magnetic storm phenomenology.

\section{Acknowledgements}

Thanks to Dr. Brian Anderson at The Johns Hopkins University Applied Physics Laboratory for computing and providing the FACs from the Iridium magnetometer data. 


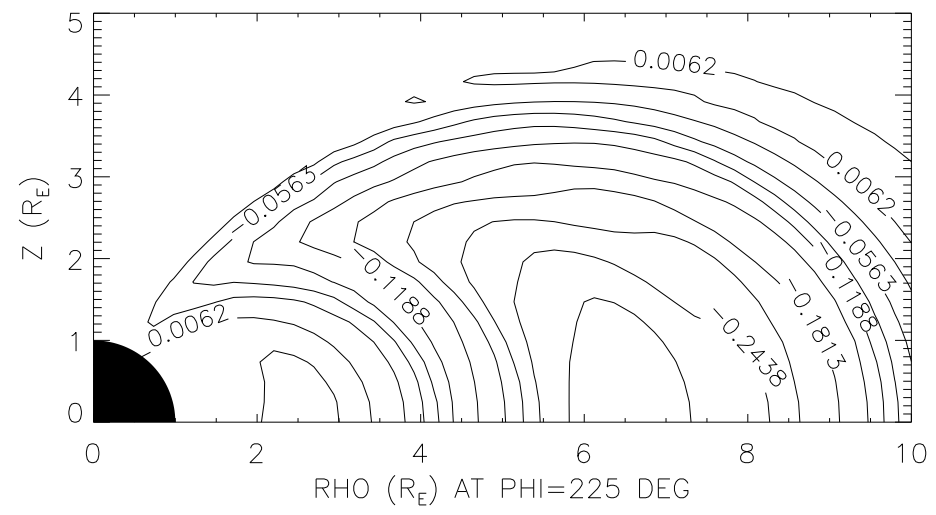

Fig. 5. Contour of the azimuthal current density in the meridional plane at 03 MLT (approximate local time of the pressure peak in Figure 1). The unit of the current density contours is $\mathrm{nA} \mathrm{m}^{-2}$.

\section{References}

C:son Brandt, P., Demajistre, R., Roelof, E. C., Mitchell, D. G., Mende, S., 2002a. IMAGE/HENA: Global ENA imaging of the plasmasheet and ring current during substorms. J. Geophys. Res. In press.

C:son Brandt, P., Ohtani, S., Mitchell, D. G., Fok, M. C., Roelof, E. C., Demajistre, R., 2002b. Global ENA observations of the storm mainphase ring current: Implications for skewed electric fields in the inner magnetosphere. Geophys. Res. Lett. In press.

Demajistre, R., Roelof, E. C., C:son Brandt, P., Mitchell, D. G., 2002. Retrieval of global magnetospheric ion distributions from high energy neutral atom (ENA) measurements by the IMAGE/HENA instrument. J. Geophys. Res. To be submitted.

Fok, M. C., Wolf, R. A., Spiro, R. W., Moore, T. E., 2001. Comprehensive computational model of Earth's ring current. J. Geophys. Res. 106 (A5), 8417-8424.

Foster, J. C., Rich, F. J., 1998. Prompt mid-latitude electric field effects during severe geomagnetic storms. J. Geophys. Res. 103 (A11), 26367-26372.

Ohtani, S., Nose, M., Rostoker, G., Singer, H., Lui, A. T. Y., Nakamura, M., 2001. Storm-substorm relationship: Contribution of the tail current to Dst. J. Geophys. Res. 106 (A10), 21199-21209.

Roelof, E. C., 1989. Remote sensing of the ring current using energetic neutral atoms. Adv. Space Res. 9 (12), 12195-12203. 